Review

\title{
DCIS Margins and Breast Conservation: MD Anderson Cancer Center Multidisciplinary Practice Guidelines and Outcomes
}

\author{
Henry M. Kuerer ${ }^{1 凶}$, Benjamin D. Smith², Mariana Chavez-MacGregor 3 , 4, Constance Albarracin ${ }^{5}$, Carlos H. \\ Barcenas $^{3}$, Lumarie Santiago ${ }^{6}$, Mary E. Edgerton ${ }^{5}$, Gaiane M. Rauch ${ }^{6}$, Sharon H. Giordano ${ }^{3,4}$, Aysegul

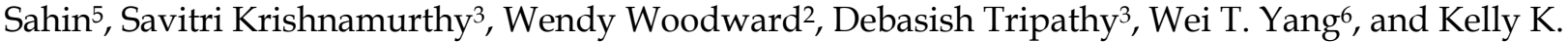 \\ Hunt ${ }^{1 凶}$
}

1. Department of Breast Surgical Oncology, The University of Texas MD Anderson Cancer Center, Houston, TX;

2. Department of Radiation Oncology, The University of Texas MD Anderson Cancer Center, Houston, TX;

3. Department of Breast Medical Oncology, The University of Texas MD Anderson Cancer Center, Houston, TX;

4. Department of Health Services Research, The University of Texas MD Anderson Cancer Center, Houston, TX;

5. Department of Pathology, The University of Texas MD Anderson Cancer Center, Houston, TX;

6. Department of Diagnostic Radiology, The University of Texas MD Anderson Cancer Center, Houston, TX

$\triangle$ Corresponding authors: Henry M. Kuerer, MD, PhD, FACS, Kelly K. Hunt, MD, FACS, Department of Breast Surgical Oncology, The University of Texas MD Anderson Cancer Center, 1400 Pressler St, Unit 1434, Houston, TX 77030 E-mail: hkuerer@mdanderson.org; khunt@mdanderson.org Telephone: 713-745-5043 Fax: 713-794-5026

(C) Ivyspring International Publisher. This is an open access article distributed under the terms of the Creative Commons Attribution (CC BY-NC) license (https://creativecommons.org/licenses/by-nc/4.0/). See http://ivyspring.com/terms for full terms and conditions.

Received: 2017.05.04; Accepted: 2017.07.14; Published: 2017.08.22

\begin{abstract}
Recent published guidelines suggest that adequate margins for DCIS should be $\geq 2 \mathrm{~mm}$ after breast conserving surgery followed by radiotherapy (RT). Many groups now use this guideline as an absolute indication for additional surgery. This article describes detailed multidisciplinary practices including extensive preoperative/intraoperative pathologic/histologic image-guided assessment of margins, offering some patients with small low/intermediate grade DCIS no RT, the use/magnitude of radiation boost tailoring to margin width, and endocrine therapy for ER-positive DCIS. Use of these protocols over the past 20-years has resulted in 10-year local recurrence rates below $5 \%$ for patients with negative margins $<2 \mathrm{~mm}$ who received $\mathrm{RT}$. Patients with margins $<2 \mathrm{~mm}$ who do not receive $\mathrm{RT}$ experience significantly higher local failure rates. Thus, there is not an absolute need to achieve wider negative surgical margins when $<2 \mathrm{~mm}$ for patients treated with RT and this should be determined by the multidisciplinary team. Utilization of these multidisciplinary treatment protocols and techniques may not be exportable and extrapolated to all hospitals, breast programs and systems as they can be complex and resource intensive.
\end{abstract}

Key words: DCIS, ductal carcinoma in situ, breast cancer, surgery, pathology, radiotherapy, margins.

\section{Introduction}

Patients with negative margins after breast conserving surgery (BCS) for ductal carcinoma in situ (DCIS) are at lower risk of local recurrence (LR) compared with patients with positive margins; however the optimal margin width has been a topic of debate for many decades. The current management of DCIS includes a wide array of treatment options in patients and physicians struggle with decision making in order to avoid overtreatment or under treatment. Ongoing trials in Europe and the United States are randomizing patients with low risk DCIS to standard care (surgery with or without radiation therapy) versus percutaneous biopsy and surveillance with surgery/radiotherapy only with progression to invasive disease. On the other hand we continue to debate what constitutes an adequate margin of resection among patients undergoing BCS followed by whole breast radiation therapy (WBRT) [1-4]. To 
complicate this further, recent meta-analyses and consensus guideline for invasive breast cancer (with and without DCIS) have defined an adequate margin of resection of no tumor on ink yet the newest consensus guideline for DCIS defines a $2 \mathrm{~mm}$ margin as the standard for an adequate margin in DCIS treated with WBRT [5-7]. Given these new developments and differences, it was of interest for our group to review MD Anderson's contemporary outcomes among patients undergoing BCS for DCIS with particular attention to our own multidisciplinary practice to establish our own guidelines as it relates to margin status in DCIS.

\section{Recent Meta-analysis and DCIS Margin Consensus Guideline}

Given the controversy and lack of consensus on what represents an adequate margin for patients with DCIS undergoing BCS, the Society of Surgical Oncology (SSO), American Society for Radiation Oncology (ASTRO), and American Society of Clinical Oncology (ASCO) convened a multidisciplinary panel to evaluate the relationship between LR and margin width [7]. The main goal of developing the guideline was to assist clinicians and patients in the decision-making process based on the best available evidence. The DCIS consensus guideline is based in a study-level meta-analysis that evaluated the effect of margin width and LR with the aim of defining a minimum negative margin to maximize local control [5].

The studies included in the meta-analysis were selected from 1,577 overall available studies. To be eligible, the study had to have at least 50 patients with DCIS undergoing BCS with WBRT, have at least 4 years of median follow-up, numerically defined margins and crude LR data available. A total of 108 studies were assessed for eligibility with 20 retrospective studies selected for inclusion. The studies selected represented 7,883 patients treated from 1968-2010. The median follow-up was 6.5 years and the median incidence of LR was $8.3 \%$. The meta-analysis included two different statistical analyses, the LR proportion was modeled using random-effects logistic meta-regression (frequentist approach) and a network meta-analysis that allowed for multiple margins distance per study (Bayesian approach). In the frequentist approach, relative to $>0$ or $1 \mathrm{~mm}$, odd ratios (ORs) for $2 \mathrm{~mm}(0.51), 3$ or $5 \mathrm{~mm}$ (0.42) and $10 \mathrm{~mm}(0.60)$ showed comparable reductions in the odds of LR. In the Bayesian analysis OR relative to positive margins for $2 \mathrm{~mm}(0.32), 3 \mathrm{~mm}$ $(0.30)$ and $10 \mathrm{~mm}(0.32)$ were similar, and were greater than the odds of LR for $>0$ or $1 \mathrm{~mm}(0.45)$. There was a lower odds of LR at $2 \mathrm{~mm}$ compared to $>0$ or $1 \mathrm{~mm}$ (relative OR 0.72) and no evidence that a distance greater than $2 \mathrm{~mm}$ had any beneficial effect. The width reported in the studies included in the meta-analysis did not allow the investigators to analyze the impact of margins 1-1.9 in the risk of LR and some groups consider this a major limitation of the study with respect to selection and utilization of a $2 \mathrm{~mm}$ guideline. Based on these results, the meta-analysis concluded the margin distances above 2 $\mathrm{mm}$ are not significantly associated with further reduction in odds of LR (3).

The SSO, ASTRO and ASCO multidisciplinary panel determined, after reviewing the best available evidence that a positive margin, defined as ink on DCIS is associated with an increased risk in LR and that such risk is not nullified by the use of WBRT. Margins of at least $2 \mathrm{~mm}$ are associated with a reduced risk or LR and margins wider that $2 \mathrm{~mm}$ are not associated with lower LR, thus the evidence does not support the routine practice of obtaining margins wider than $2 \mathrm{~mm}$ [7]. The consensus also evaluated data on endocrine therapy, radiation therapy and patient and tumor characteristics. While endocrine therapy reduces breast adverse outcomes, there is no association between therapy and margins, similarly the details associated with the dose, frequency and boost should not be dependent of margin status. The guideline multidisciplinary panel recognized that there are a number of factors associated with the risk of LR including histologic pattern, comedonecrosis, size of DCIS, and even gene expression profiles. Currently there are no data addressing whether recommended margin width should be influenced by these factors.

An area of specific clinical interest that the guideline addresses is the common scenario of patients with DCIS and microinvasion (defined as no invasive focus larger than $1 \mathrm{~mm}$ ), in this cases, based on expert opinion, the panel considered that the DCIS margin guideline should be used given that the majority of the lesion was DCIS and that the systemic management of these patients resembles that of DCIS and not invasive carcinomas. Conversely, among patients with invasive carcinoma with a DCIS component, the recommendation is to follow the invasive cancer guideline [6], given that the natural history and treatment of these lesions is more similar to invasive cancers where majority of patients receive systemic therapy. The panel noted in some cases, where an extensive intraductal component is seen, a post-excision mammography could be needed and some patients may require re-excision.

The SSO, ASTRO and ASCO guideline for DCIS margins was designed to help clinicians and patients in the decision-making process while acknowledging 
that each individual case requires clinical judgment. The authors emphasize that the guideline only applies to patients with DCIS treated with BCS and WBRT, and the recommendations therefore cannot be extrapolated to patients treated with accelerated partial breast irradiation. Although the consensus guideline clearly states that clinical judgment should be used in determining the need for further surgery in patients with negative margins $<2 \mathrm{~mm}$, clinicians and multidisciplinary teams are beginning to utilize this as an absolute indication for re-operative surgery.

\section{International guidelines for DCIS margins for BCS}

The new SSO, ASTRO, and ASCO guidelines are also consistent with the European Society for Medical Oncology (ESMO), the National Institute for Health and Clinical Excellence (NICE), and the New Zealand Guidelines Group which all define an appropriate margin width in DCIS to be $2 \mathrm{~mm}$ [8-10]. The National Comprehensive Cancer Network guidelines now also state that margins of at least $2 \mathrm{~mm}$ are associated with a reduced risk of ipsilateral breast tumor recurrence (IBTR) relative to narrower negative margin widths in patients receiving WBRT [11]. The American Society of Breast Surgeons defines a negative margin in patients with DCIS as no ink on tumor [12]. Therefore, our group was particularly interested in defining the absolute risk of local recurrence in contemporary patients with less than $2 \mathrm{~mm}$ margins treated for DCIS with BCS with and without radiotherapy at MD Anderson.

\section{Anderson contemporary outcomes of BCS for DCIS and margin width}

For patients undergoing BCS, a previous analysis of 1,216 patients treated at MD Anderson showed that the presence of close margins less than 2 $\mathrm{mm}$ did not increase the patient's risk for in-breast tumor recurrence (IBTR) in comparison to more widely negative margins, provided the patient also received radiation therapy [13]. Specifically, risk of IBTR at 5-years was 3.7\% among women with widely negative margins $\geq 2 \mathrm{~mm}$ compared to $2.2 \%$ among women with close or positive margins. Approximately $80 \%$ of patients in this cohort received radiation therapy following breast conserving surgery. In multivariable analysis, age, receipt of radiation therapy, and size of DCIS correlated with IBTR risk, whereas margin status did not.

We recently updated this cohort with additional patients and longer follow-up and analyzed outcomes. We identified 1,491 patients with DCIS undergoing definitive BCS from 1996 to 2010 [14, 15].
Cox proportional hazard models were used to examine the relationship between margin width $(<2$ $\mathrm{mm}$ or $\geq 2 \mathrm{~mm}$ ) and local - regional recurrence (LRR) by receipt of adjuvant radiation therapy (RT). Patients with positive margins defined as ink on tumor were excluded and the median follow-up of the cohort was 8.7 years. Univariate analysis of age, family history, grade, tumor size, presence of comedonecrosis, RT, adjuvant hormonal therapy, ER status, and margin width revealed younger age $(<40 \mathrm{yr})$, no RT, and margin width $<2 \mathrm{~mm}(\mathrm{n}=100)$ to be significantly associated with LRR. The association between margin width and LRR significantly differed by receipt of adjuvant radiation therapy. There was no statistical significant difference in LRR for patients with margins $<2 \mathrm{~mm}$ vs $\geq 2 \mathrm{~mm}$ who received RT, (10-year LRR $4.8 \%$ vs $3.3 \%$, respectively; $\mathrm{p}=0.72$; Figure 1a). For patients who did not receive RT, those with margins $<2 \mathrm{~mm}$ were significantly more likely to develop a LRR than those with margins $\geq 2 \mathrm{~mm}$ (10-year LRR $30.9 \%$ vs. $5.4 \%$, respectively; $p=0.003$; Figure 1b). Thus, in our contemporary multidisciplinary practice, for patients with $<2 \mathrm{~mm}$ margins receiving adjuvant radiation therapy, there is no detectable significant difference in locoregional recurrence when compared to patients with $\geq 2 \mathrm{~mm}$ margins. In this regard, it is also interesting to note that one of the other largest studies evaluating the relationship between margin width and recurrence of DCIS from investigators at the Memorial Sloan Kettering Cancer Center did not identify a significant association of recurrence with margin width of $\leq 2$ $\mathrm{mm}$ compared with larger margins for patients receiving radiotherapy [16]. Therefore in general, at MD Anderson additional surgery for wider margins of resection are not routinely justified in this group of patients but should be obtained for patients with $<2$ $\mathrm{mm}$ margins who are not plan to receive radiotherapy. Important caveats for this recommendation do not include patients who have multiple very close margins or evidence of residual malignant appearing calcifications on mammography. These patients do require additional excision. Thus, although it has been well known that reexcision after surgery for DCIS with close margins after conservative surgery will find additional disease in a significant proportion of patients [17], the use of radiotherapy in this scenario appears to abrogate the long-term risk of recurrence.

\section{Anderson multidisciplinary practices facilitating negative margins and local control of disease}

In our practice there is extensive interaction between the radiologist, the surgeon, and pathologists 
preoperatively, intraoperatively, and following surgery to ensure adequate resection of the lesion to minimize the need for repeat surgery and LRR. Decisions regarding adequate extent of margins, use of radiation and/or endocrine therapy are made in a multidisciplinary manner and patient input with respect to perceived benefit and risk is also of prime importance.

\section{Breast imaging and DCIS}

\section{Screening}

Mammography is the main modality for detection and determination of the extent of DCIS particularly when presenting as calcifications with a reported sensitivity of $87-95 \%$ [18-21]. Although the sensitivity of breast ultrasound (47-71.3\%) is lower than mammography, ultrasound can be useful as an adjuvant imaging modality when the disease is non-calcified, occult by mammography, or in symptomatic patients [22, 23]. It also can identify a mass lesion that may represent an invasive component.

\section{Determination of disease extent and biopsy}

Breast MRI has high sensitivity for DCIS detection particularly in higher grade disease (73-100\%) [24, 25]. Breast MRI may be helpful when disease has an atypical presentation and in the subset of micropapillary DCIS where mammography and sonography can underestimate the extent of disease [26-28]. Breast MRI has not been proven to improve selection of patients for margin-negative breast conserving therapy. Further, the use of breast MRI in patients with pure DCIS has not impacted long term outcome following breast conserving therapy [29]. Therefore, breast MRI is not routinely utilized for patients with DCIS at our institution.

Defining the extent of malignant appearing calcifications helps the surgical team to determine which patients would be optimal candidates for breast conserving therapy given the breast to tumor size ratio. A recent evaluation of breast microcalcifications among 1657 patients with pure DCIS treated at MD Anderson, identified that fine linear (branching) microcalcifications were associated with a 5.2 fold increase in LRR. Further, extremely dense breast tissue was significantly associated with dense breasts and biopsy-proved multicentric disease, and such patients were more likely to undergo mastectomy compared with older patients [30]. It is also interesting to note that ER-negative DCIS is more frequently visible on sonography than ER-positive DCIS.[19] For lesions that have extensive malignant appearing calcifications the extremes of the lesion are biopsied to pathologically confirm the extent of disease and mark the area for potential surgical excision. If the disease is multicentric, mastectomy is generally performed although oncoplastic
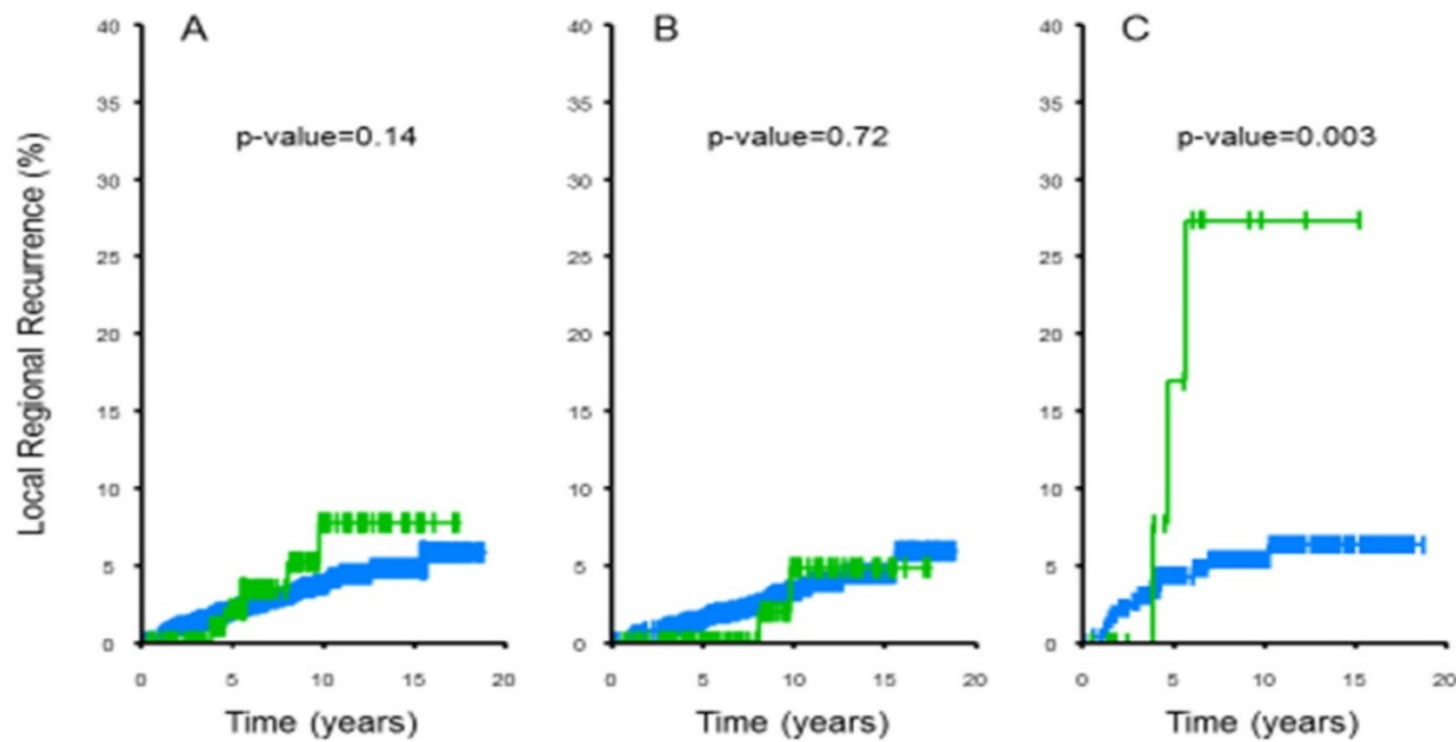

Figure 1. Local-regional recurrence outcome among 1,491 patients with DCIS treated with breast conservation all patients (A), with (B) and without (C) radiotherapy, stratified by margin status at MD Anderson Cancer Center 1996-2010. There was no statistical significant difference in LRR for patients with margins $<2 \mathrm{~mm}$ vs $\geq 2 \mathrm{~mm}$ who received RT, (10-year LRR $4.8 \%$ vs $3.3 \%$, respectively; $\mathrm{P}=0.72$; B). For patients who did not receive RT, those with margins $<2 \mathrm{~mm}$ were significantly more likely to develop a LRR than those with margins $\geq 2 \mathrm{~mm}(10$-year LRR $30.9 \%$ vs. $5.4 \%$, respectively; $\mathrm{P}=0.003$; C) [14, 15]; with permission from Annals of Surgery. Promotional and commercial use of the material in print, digital or mobile device format is prohibited without permission from the publisher Wolters Kluwer. Please contact healthpermissions@wolterskluwer.com for further information. 
reconstruction can facilitate breast conserving procedures in patients with larger breasts. When a decision has been made that the patient is an acceptable candidate for BCS, the lesion(s) in question are routinely localized with wire(s) or with a radioactive seed(s), depending on surgeon preference. Extensive interaction with the radiologist, the surgeon, and pathologists preoperatively, intraoperatively, and following surgery helps to ensure adequate resection. As noted above, when there is a margin less than $2 \mathrm{~mm}$, it is our general practice to perform a postoperative diagnostic mammogram to ensure that no suspicious-appearing residual calcifications remain. If there is any question regarding the malignant nature of these calcifications, they can be biopsied under stereotactic guidance to determine if reexcision is indicated. If the biopsy reveals residual ductal carcinoma, the calcification should be localized prior to the time of reexcision to assure removal.

\section{Surgical Technique and Considerations}

During BCS, it is not routine to excise skin at the time of surgical resection for DCIS at MD Anderson. The practice of excision of the skin to the pectoralis fascia has its origins from the 1970s and 80 s as a routine procedure for quadrantectomy and among early surgical practitioners in the United States. There is no need to remove the skin for noninvasive breast cancer except if there are extensive malignant appearing microcalcifications just beneath the skin seen on preoperative imaging, intraoperative imaging, or as part of a planned oncoplastic procedure. In general, the breast surgeons at MD Anderson have largely moved to localizations utilizing radioactive seeds (one or more as needed) since the seeds can be placed on a day remote from the operative procedure allowing more flexibility in the OR schedule. We are also performing prospective clinical trials evaluating newer technologies for intraoperative localization. Our practice for the last 25 years has involved extensive intraoperative specimen imaging and pathology evaluation to ensure complete resection and negative margins, described below in the pathologic processing section of this manuscript.

We do not routinely obtain cavity shave margins as this is believed to adversely affect cosmetic outcome by removing additional normal breast tissue that is uninvolved by DCIS [31]. Instead we perform a targeted resection of any suspicious margins based on the intraoperative assessment by imaging and pathology. Utilizing selective frozen section analysis of close or suspicious margins identified on the intraoperative images allows for the $\sim 35 \%$ of cases to move from positive intraoperative margins to negative final pathologic margins for DCIS [31]. Our group is also testing the potential of high-resolution 3D tomosynthesis for intraoperative specimen imaging evaluation for potential increased efficiency. Radiopaque clips are placed routinely in the resection cavity to facilitate identification of the area for radiation therapy and to facilitate the use of a boost when indicated. Although the median size of DCIS cases treated with BCS over the past 20 years at MD Anderson is only $1 \mathrm{~cm}$, during the same time period the use of oncoplastic surgery has allowed for an increasing number of patients with an unfavorable tumor location to become BCS-eligible [14, 32]. Essentially, if all malignant appearing calcifications can be removed utilizing this approach and the margins are negative, there is no absolute size criteria utilized at our institution for the selection of patients for BCS in the treatment of DCIS. This approach, however, sometimes makes delivery of a radiation boost complex. While some surgeons have advocated for mastectomy if a positive margin is obtained after an oncoplastic surgery, our group evaluates each case and does not feel that mastectomy is routinely needed. The segmental cavity can be easily identified and specific margins can be excised to achieve negative margins with re-operative surgery. When re-excisions are needed following BCS with oncoplastic reconstruction, the procedures are performed jointly with the plastic surgeons and breast surgical oncologist. Lymphatic mapping with sentinel lymph node biopsy is considered on a case by case basis depending on the size of the DCIS lesion and the complexity of the oncoplastic reconstruction [33-35]. Sentinel lymph node biopsy may be performed when there is a large size of DCIS where upstaging to invasive disease approaches $25 \%$ or if there is suspicion for invasion on imaging or the diagnostic biopsy. The development of various technologies to identify positive margins during surgery for invasive and in situ disease is a burgeoning area of research and business development. At this time, there is no unequivocal evidence that these technologies can more accurately predict and identify positive margins for DCIS compared with other standard processes. This area remains an important area of research intended to decrease the need for reoperative surgery which can impact cosmetic outcomes and quality of life.

\section{Pathologic processing of DCIS}

The strongest predictor of local recurrence of DCIS after BCS is a positive surgical margin at the time of initial surgery. Achieving negative margins for DCIS can be quite challenging, as most DCIS lesions are non-palpable and are usually detected 
only by radiologic findings as microcalcifications [30, 36]. Accurate assessment of the extent of DCIS and the margin status of BCS specimens is often difficult and the handling of these specimens requires special care [37-39]. Use of specimen radiographs is strongly recommended [37, 40]. Orientation of the BCS specimens by the surgeon is crucial to assure correct orientation and multiple color inking is routinely utilized at MD Anderson to facilitate either intraoperative or postoperative targeted excision for margin control. This method also facilitates measurement of the distance to each margin more precisely. Margins are first evaluated on specimen radiographs to ensure that all radiographic abnormalities are removed and margins are free of any mammographic abnormalities (Figure 2). Intra-operative specimen imaging is performed by digital imaging in the pathology suite located in the operating room. The images are then viewed by the pathologist, the surgeon on monitors in the operating room, and radiologists concurrently on monitors in the radiology suite. This permits real-time multidisciplinary communication to assist with directed re-excisions if necessary. Sections are then submitted for histologic evaluation. Perpendicular sections are recommended for areas where the margins appear to be close, as they allow accurate measurement of the distance to the margin [41]. In general, more extensive sampling is necessary for DCIS samples than for invasive lesions which tend to be more macroscopically evident. Pathology reports should include details of the closest measurement to each margin and, for involved margins, the extent of the involvement can be categorized as focal or extensive. Adequacy of margins should be evaluated for each patient and the decision for reoperative surgery should be based on additional factors such as histologic type, grade and presence or absence of any residual mammographic abnormality.

\section{Radiation Therapy for DCIS}

Use of RT is a key consideration when evaluating the adequacy of resection for DCIS. At MD Anderson, we have used a tailored approach when considering the radiation boost dose based on the final surgical margin status. The boost dose is 16 Gy in 8 fractions for patients with positive margins, $14 \mathrm{~Gy}$ in 7 fractions for patients with close margins $<2 \mathrm{~mm}$, and $10 \mathrm{~Gy}$ in 5 fractions for patients with margins $>2 \mathrm{~mm}$. While there are no high quality prospective data to document appropriate boost dosing and indications in DCIS, the favorable results from the MD Anderson DCIS series cited above support this tailored approach [42]. Further, for patients with DCIS, the extremely favorable results of the Radiation Therapy and Oncology Group (RTOG) 9804 clinical trial, in which 7-year IBTR risk was less than 1\% among patients treated with WBRT without a boost, present a strong argument that a tumor bed boost is not needed in the patient population of low-risk DCIS [43].

Recently, there has been a growing body of data illustrating that the Breast DCIS score, developed and marketed by Genomic Health, can prognosticate 10-year risk of IBTR (invasive and in situ events) and 10-year risk of invasive IBTR in patients treated with BCS without RT. Based on its value, proponents have argued that this test may be used to predict which patients will benefit from RT and which patients do not require it. However, it should be noted that the test itself has only been validated as a prognostic test and not a predictive test. Further, a recently published decision analysis suggested that routine incorporation of the test into clinical practice was unlikely to meet societal thresholds for cost effectiveness [44].

With these limitations acknowledged, our group participated in a multicenter study that found that the test may have certain clinical utility [45]. For example, in a patient who meets RTOG 9804 criteria for low risk DCIS (size $\leq 2.5 \mathrm{~cm}$, mammographically detected, and low to intermediate grade), the estimated risk of IBTR is $1 \%$ /year treated without radiotherapy based on this trial data. If the patient is interested in observation, then the Breast DCIS Score could be used as a confirmatory test to "rule-in" the decision for omission of radiation therapy after BCS. This "rule-in" observation approach can be particularly helpful in patients who may be considered borderline candidates for omission of radiation solely based on clinical grounds - for example a very young patient with otherwise favorable DCIS, or a patient with low to intermediate grade DCIS who has evidence of comedonecrosis on pathology. Finally, for patients perceived to be at higher risk for IBTR based solely on clinical-pathologic grounds but who nevertheless are strongly motivated to avoid radiation, the Breast DCIS Score could be useful to "rule-out" a decision for omission of radiation and provide more compelling data for the patient to justify the decision for treatment. We expect that indications for use of the Breast DCIS Score will continue to be refined as additional data become available.

Consistent with data from MD Anderson, a study from Memorial Sloan Kettering Cancer Center sought to estimate the relative benefit of RT following BCS as a function of margin status [46]. In this study, margin status was categorized as $<1 \mathrm{~mm}, 1$ to $9 \mathrm{~mm}$, or $\geq 10 \mathrm{~mm}$. Among patients who received RT following BCS, 10-year risk of IBTR was nearly identical regardless of margin status and ranged from $11-13 \%$. In contrast, among patients who did not 
receive radiation therapy, 10-year risk of IBTR was $41 \%$ for those with close margins $<1 \mathrm{~mm}, 27 \%$ for those with negative margins ranging from 1 to $9 \mathrm{~mm}$, and $21 \%$ for those with margins $>10 \mathrm{~mm}$. The conclusion from these important studies is that when patients undergo BCS at large centers with careful assessment of pathologic margins and multidisciplinary decision making, a close margin is not an adverse risk factor for LR provided patients receive appropriate WBRT and there is documentation of removal of malignant appearing calcifications. a)

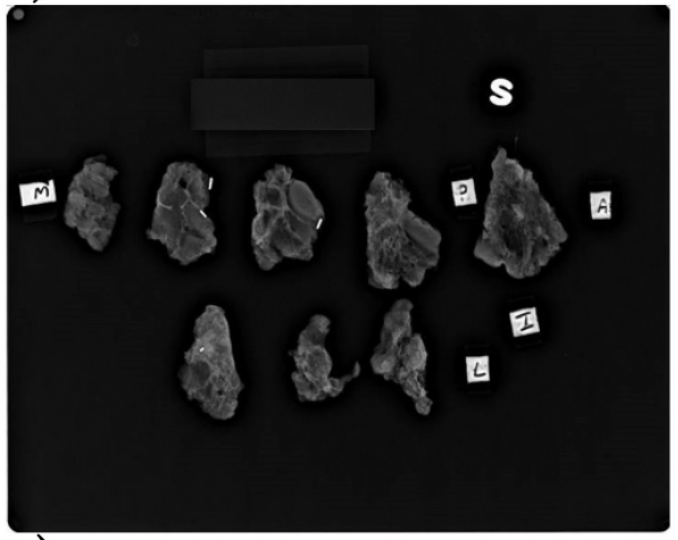

c)

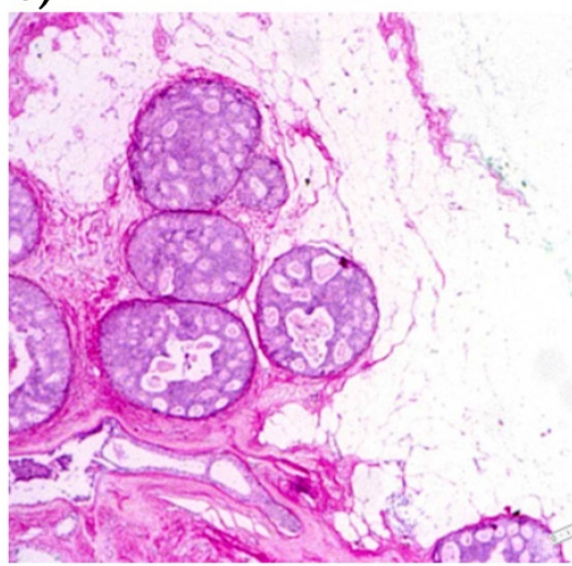

b)

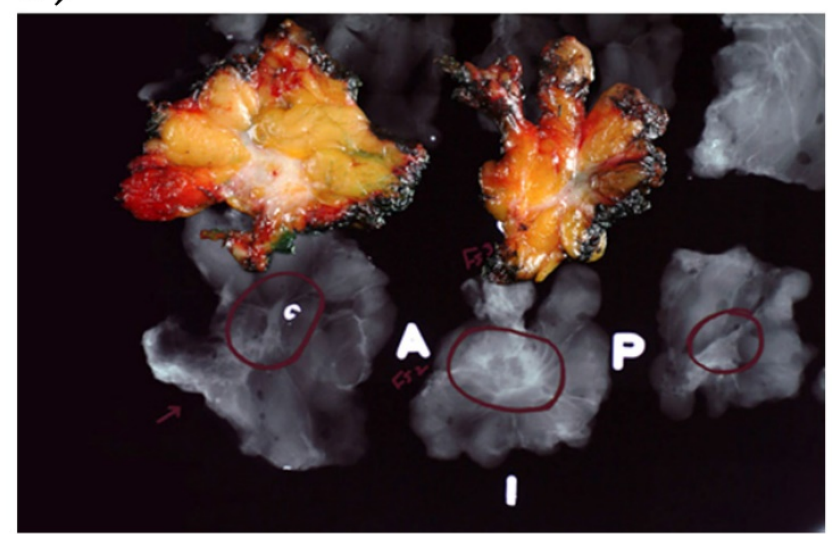

d)

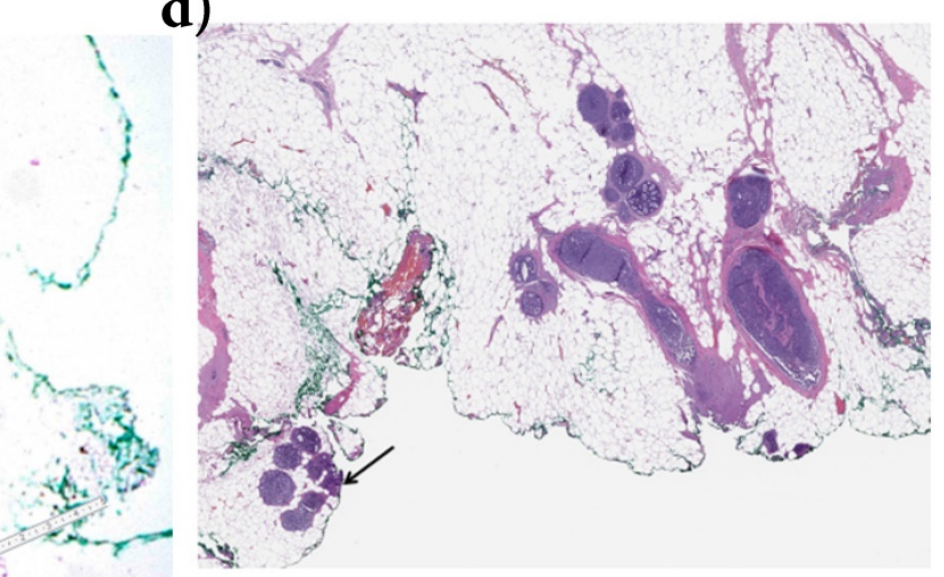

e)

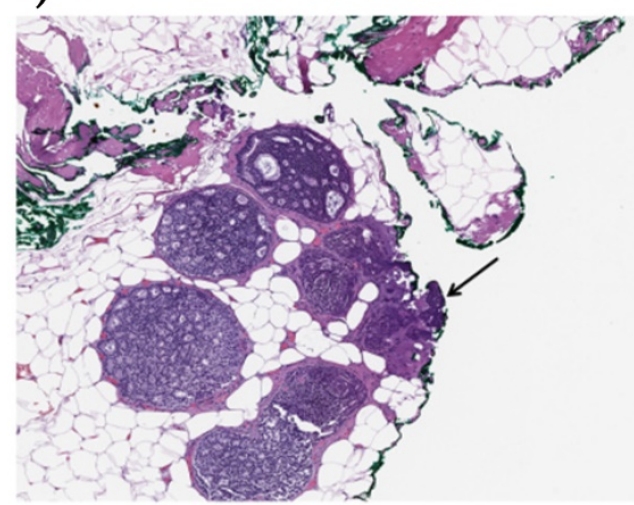

Figure 2. MD Anderson intraoperative pathologic and radiologic processing of segmental mastectomy specimens for DCIS a) Intraoperative specimen radiograph of the sectioned specimen is very important in the evaluation of margins. Careful evaluation of these slices helps to identify the targeted lesions as well as other abnormalities. Additional margins can be obtained when the abnormality is present close to or at the margins and each slice is designated as superior, inferior, medial, lateral, anterior, and posterior based on placement of the slices. The area on the $2^{\text {nd }}$ row first slice on the left was interpreted as suspicious for residual DCIS and additional margin was recommended. b) Correlation of gross appearance and specimen radiograph is an important aspect of intraoperative evaluation of breast specimens. The gray white area present on gross inspection corresponds to architectural distortion/density on specimen radiograph (circled area). c) DCIS extending within $2.5 \mathrm{~mm}$ to an inked margin. The distance between green ink and ductal carcinoma in situ is reported as the margin width (lower right corner). d) DCIS is focally extending to margin (arrow). e) Higher magnification showing ducts with in situ carcinoma cells extending to inked margin (arrow). 
Table 1. Randomized controlled trials of endocrine therapy in patients with DCIS

\begin{tabular}{|c|c|c|c|c|}
\hline Clinical trial & Eligibility & Sample size & Comparison arms & Results \\
\hline NSABP B-24[47] & Lumpectomy and radiation therapy & 1804 & Tamoxifen vs. placebo & $\begin{array}{l}\text { Breast cancer events at } 5 \text { years: } 8.2 \% \text { (Tamoxifen) vs. } \\
13.4 \% \text { (placebo); } p=0.0009\end{array}$ \\
\hline $\begin{array}{l}\text { NSABP B-24 with ER and } \\
\text { PR assessment[48] }\end{array}$ & Lumpectomy and radiation therapy & 732 & Tamoxifen vs. placebo & $\begin{array}{l}\text { In patients with ER+ DCIS, tamoxifen decreased } \\
\text { breast cancer events at } 10 \text { years; HR: } 0.64,95 \% \text { CI: } \\
0.48-0.86 \text {; no benefit seen in ER-negative DCIS }\end{array}$ \\
\hline UK/ANZ DCIS[50] & $\begin{array}{l}\text { Completely locally excised DCIS } \\
\text { (negative margins) }\end{array}$ & 1701 & $\begin{array}{l}2 \times 2 \text { factorial of } \\
\text { tamoxifen, or } \\
\text { radiotherapy or both }\end{array}$ & $\begin{array}{l}\text { Median follow-up of } 12.7 \text { years, tamoxifen reduced } \\
\text { the incidence of all breast events; HR: } 0.71,95 \% \text { CI: } \\
0.58-0.88 \text {; benefit mainly among those who did not } \\
\text { receive radiotherapy }\end{array}$ \\
\hline NSABP B-35[53] & $\begin{array}{l}\text { Postmenopausal, } \\
\text { hormone-receptor-positive DCIS who } \\
\text { underwent lumpectomy with clear } \\
\text { margins }\end{array}$ & 3104 & $\begin{array}{l}\text { Anastrozole vs. } \\
\text { tamoxifen }\end{array}$ & $\begin{array}{l}\text { Median follow-up of } 9 \text { years, } 122 \text { breast cancer-free } \\
\text { events in the tamoxifen group vs. } 90 \text { in the } \\
\text { anastrozole group; HR: } 0.73,95 \% \text { CI: } 0.56-0.96 \text {; benefit } \\
\text { seen only in patients younger than } 60 \text { years of age }\end{array}$ \\
\hline IBIS-II DCIS[55] & $\begin{array}{l}\text { Postmenopausal, } \\
\text { hormone-receptor-positive DCIS who } \\
\text { underwent lumpectomy with clear } \\
\text { margins }\end{array}$ & 2980 & $\begin{array}{l}\text { Anastrozole vs. } \\
\text { tamoxifen }\end{array}$ & $\begin{array}{l}\text { Median follow-up of } 7.2 \text { years, } 67 \text { recurrences for } \\
\text { anastrozole vs. } 77 \text { for tamoxifen; HR: } 0.89,95 \% \text { CI: } \\
0.64-1.23 \text {; no differences in outcomes between } \\
\text { treatments }\end{array}$ \\
\hline
\end{tabular}

Abbreviations: NSABP: National Surgical Adjuvant Breast and Bowel Project; UK/ANZ: United Kingdom, Australia and New Zealand; IBIS: International Breast Cancer Intervention Study; HR: Hazard ratio; CI: confidence interval

\section{Endocrine therapy following BCS for DCIS}

The effectiveness of tamoxifen in the adjuvant treatment of DCIS patients was first demonstrated when the results of the NSABP B-24 clinical trial were published in 1999 [47]. This double-blinded, randomized, controlled clinical trial enrolled 1804 women with DCIS who underwent BCS and WBRT therapy and were randomized to receive tamoxifen versus placebo. Patients who received tamoxifen had fewer ipsilateral and contralateral breast cancer events. Later, it was demonstrated that the benefit was largely seen in the subset of patients who had ER-positive DCIS [48]. Interestingly, $25 \%$ of the participants of B-24 had positive or unknown surgical margins after surgery, and the 15-year IBTR rate in the placebo cohort was $17.4 \%$ among patients with positive margins versus $7.4 \%$ in patients with negative margins. Tamoxifen lowered the IBTR rate to $11.5 \%$ in those with positive margins, while it had no effect among the group of patients with negative surgical margins (7.4\% vs. $7.5 \%)$ [49]. In the UK/ANZ DCIS trial, which enrolled 1701 patients who had undergone local excision achieving negative surgical margins, tamoxifen reduced the incidence of recurrent ipsilateral DCIS and contralateral tumors; however, the benefit of tamoxifen was mainly seen among the cohort of patients who did not receive RT [50, 51]. In the meantime, the aromatase inhibitors have shown superiority to tamoxifen in the treatment of postmenopausal women with hormone receptorpositive invasive breast cancer [52]. Subsequently, two large, double-blinded, randomized clinical trials compared anastrozole versus tamoxifen in postmenopausal women with hormone receptorpositive DCIS. NSABP B-35, which enrolled 3104 patients who underwent BCS with clear margins, showed that anastrozole use was associated with an improved breast cancer-free interval compared to tamoxifen, mainly among those with an age younger than 60 years [53]. This study also showed that there were important differences in patient reported outcomes between both therapeutic options [54]. The IBIS-II DCIS clinical trial, which enrolled 2980 postmenopausal women who had clear margins after BCS, demonstrated non-inferiority for anastrozole and no clear efficacy differences between both treatments. Although adherence to treatment was relatively low and similar among both treatment arms $(67 \%)$, there were significant differences in adverse events, where patients who received anastrozole had more musculoskeletal events including fractures and osteoporosis, while those who received tamoxifen had more vasomotor, gynecological and thromboembolic events [55]. The risk of death from breast cancer after a diagnosis of DCIS is similar compared to a healthy age-adjusted population; however, young age at diagnosis and a black race may be associated with increased risk [56].

In summary, the use of endocrine therapy in the adjuvant treatment of DCIS reduces breast cancer events, but the absolute benefit is small, and there appears to be minimal benefit when the surgical margins are negative and the patients receive RT. The benefits of endocrine therapy for ipsilateral recurrence and for new contralateral breast cancer are similar. At MD Anderson, the use of endocrine therapy is discussed for all patients with ER-positive DCIS receiving BCS with respect to potential benefit and side effects. Initially, at MD Anderson as well as within the National Comprehensive Cancer Network, endocrine therapy use and adherence was low [57, 58]. In our updated analysis, approximately $57 \%$ of patients with ER-positive DCIS take endocrine therapy following BCS for DCIS [14]. The choice of 
specific endocrine therapy in postmenopausal women with DCIS is tailored according to the side effect profile and patient preference.

\section{Conclusions}

The management of DCIS requires multidisciplinary involvement from screening and diagnosis to therapy and survivorship. We are in a unique time where ongoing clinical trials for low and intermediate grade ductal carcinoma in situ in Europe and the United States are now randomizing patients to surveillance after percutaneous biopsy alone with surgery or radiation only on progression to invasive disease versus guideline concordant care with or without RT and endocrine therapy. Margin status in patients undergoing BCS for DCIS has been studied extensively and remains controversial. Meta-analyses derived from older retrospective studies may not be broadly applicable to all patients treated today in all facilities. Improvements in imaging and accepted protocols for pathologic evaluation of margins, in addition to innovations in RT targeting and dosing have significantly changed the outcomes for patients with DCIS. Our data regarding intraoperative evaluation of margin status may not be applicable to groups who do not employ extensive pathologic evaluation of DCIS lesions during surgery. Notwithstanding, it is clear that patients with positive margins after BCS have an increased risk of persistence/recurrence of disease and should be offered additional re-excision. For patients with no DCIS at the inked margin who receive RT, there is no significant increased risk of local recurrence at 10 years follow-up. For patients with close margins $(<2$ $\mathrm{mm}$ ) not receiving radiotherapy, local recurrence is significant and we recommend re-excision for wider negative margins.

\section{Acknowledgement}

This work was supported by the PH and Fay Etta Robinson Distinguished Professorship in Cancer Research (HMK), the Hamill Foundation Distinguished Professorship in honor of Richard G. Martin, Sr. (KKH), and a Cancer Center Support Grant from the National Institutes of Health (NIH) (CA16672).

\section{Competing Interests}

The authors have declared that no competing interest exists.

\section{References}

1. Lari SA, Kuerer HM. Biological Markers in DCIS and Risk of Breast Recurrence: A Systematic Review. J Cancer. 2011; 2: 232-61.

2. Kuerer HM. Ductal carcinoma in situ: treatment or active surveillance? Expert Rev Anticancer Ther. 2015; 15: 777-85.
3. Francis A, Thomas J, Fallowfield L, Wallis M, Bartlett JM, Brookes C, et al. Addressing overtreatment of screen detected DCIS; the LORIS trial. Eur J Cancer. 2015; 51: 2296-303.

4. Kuerer HM, Albarracin CT, Yang WT, Cardiff RD, Brewster AM, Symmans $\mathrm{WF}$, et al. Ductal carcinoma in situ: state of the science and roadmap to advance the field. J Clin Oncol. 2009; 27: 279-88.

5. Marinovich ML, Azizi L, Macaskill P, Irwig L, Morrow M, Solin LJ, et al. The Association of Surgical Margins and Local Recurrence in Women with Ductal Carcinoma In situ Treated with Breast-Conserving Therapy: A Meta-Analysis. Ann Surg Oncol. 2016; 23: 3811-21.

6. Moran MS, Schnitt SJ, Giuliano AE, Harris JR, Khan SA, Horton J, et al. Society of Surgical Oncology-American Society for Radiation Oncology consensus guideline on margins for breast-conserving surgery with whole-breast irradiation in stages I and II invasive breast cancer. Ann Surg Oncol. 2014; 21: 704-16.

7. Morrow M, Van Zee KJ, Solin LJ, Houssami N, Chavez-MacGregor M, Harris $\mathrm{JR}$, et al. Society of Surgical Oncology-American Society for Radiation Oncology-American Society of Clinical Oncology Consensus Guideline on Margins for Breast-Conserving Surgery with Whole-Breast Irradiation in Ductal Carcinoma In situ. Ann Surg Oncol. 2016; 23: 3801-10.

8. Senkus E, Kyriakides S, Ohno S, Penault-Llorca F, Poortmans P, Rutgers E, et al. Primary breast cancer: ESMO Clinical Practice Guidelines for diagnosis, treatment and follow-up. Ann Oncol. 2015; 26 Suppl 5: v8-30.

9. National Institute of Health and Clinical Excellence. Early and locally advanced breast cancer: diagnosis and treatment. NICE clinical guideline 80 . Surgery to the Breast (1.31). https://wwwniceorguk/guidance/cg80/ chapter/1-Guidance\#surgery-to-the-breast. 2009.

10. New Zealand Guidelines Group (NZGG). Ductal carcinoma in situ. Management of early breast cancer. Wellington: New Zealand Guidelines Group (NZGG). 2009: 133-41.

11. National Comprehensive Cancer Network (NCCN). NCCN clinical practice guidelines; Breast Cancer. Version 1.2017. www.NCCN.org.

12. American Society of Breast Surgeons. The American Society of Breast Surgeons Position Statement on Breast Cancer Lumpectomy Margins. https://www.breastsurgeons.org/new_layout/about/statements/PDF_State ments/Lumpectomy_Margins.pdf.

13. Alvarado R, Lari SA, Roses RE, Smith BD, Yang W, Mittendorf EA, et al. Biology, treatment, and outcome in very young and older women with DCIS. Ann Surg Oncol. 2012; 19: 3777-84.

14. Tadros AB, Smith BD, Shen Y, Lin H, Krishnamurthy S, Lucci A, et al. Contemporary breast conservation patient outcomes for ductal carcinoma in situ and margins $<2 \mathrm{~mm}$. Proceedings of the American Society of Clinical Oncology; 2017. http://abstracts.asco.org/.

15. Tadros AB, Smith BD, Shen Y, Lin H, Krishnamurthy S, Lucci A, et al. Ductal Carcinoma in situ and Margins <2 mm: Contemporary Outcomes with Breast Conservation. Ann Surg. 2017; doi: 10.1097/SLA.0000000000002439. [Epub ahead of print]

16. Van Zee KI, Subhedar P, Olcese C, Patil S, Morrow M. Relationship Between Margin Width and Recurrence of Ductal Carcinoma In situ: Analysis of 2996 Women Treated with Breast-conserving Surgery for 30 Years. Ann Surg. 2015; 262: 623-31.

17. Merrill AL, Tang R, Plichta JK, Rai U, Coopey SB, McEvoy MP, et al. Should New "No Ink On Tumor" Lumpectomy Margin Guidelines be Applied to Ductal Carcinoma In situ (DCIS)? A Retrospective Review Using Shaved Cavity Margins. Ann Surg Oncol. 2016; 23: 3453-8.

18. Evans A, Pinder S, Wilson R, Sibbering M, Poller D, Elston C, et al. Ductal carcinoma in situ of the breast: correlation between mammographic and pathologic findings. AJR American journal of roentgenology. 1994; 162: 1307-11.

19. Scoggins ME, Fox PS, Kuerer HM, Rauch GM, Benveniste AP, Park YM, et al. Correlation between sonographic findings and clinicopathologic and biologic features of pure ductal carcinoma in situ in 691 patients. AJR American journal of roentgenology. 2015; 204: 878-88.

20. Wright B, Shumak R. Part II. Medical imaging of ductal carcinoma in situ. Current problems in cancer. 2000; 24: 112-24.

21. Yang WT, Tse GM. Sonographic, mammographic, and histopathologic correlation of symptomatic ductal carcinoma in situ. AJR American journal of roentgenology. 2004; 182: 101-10.

22. Jin ZQ, Lin MY, Hao WQ, Jiang HT, Zhang $\mathrm{L}, \mathrm{Hu} W H$, et al. Diagnostic evaluation of ductal carcinoma in situ of the breast: ultrasonographic, mammographic and histopathologic correlations. Ultrasound in medicine \& biology. 2015; 41: 47-55.

23. Wang LC, Sullivan M, Du H, Feldman MI, Mendelson EB. US appearance of ductal carcinoma in situ. Radiographics: a review publication of the Radiological Society of North America, Inc. 2013; 33: 213-28.

24. Greenwood HI, Heller SL, Kim S, Sigmund EE, Shaylor SD, Moy L. Ductal carcinoma in situ of the breasts: review of MR imaging features. Radiographics: a review publication of the Radiological Society of North America, Inc. 2013; 33: 1569-88

25. Kuhl CK, Schrading S, Bieling HB, Wardelmann E, Leutner CC, Koenig R, et al. MRI for diagnosis of pure ductal carcinoma in situ: a prospective observational study. Lancet (London, England). 2007; 370: 485-92.

26. Berg WA, Gutierrez L, NessAiver MS, Carter WB, Bhargavan M, Lewis RS, et al. Diagnostic accuracy of mammography, clinical examination, US, and MR 
imaging in preoperative assessment of breast cancer. Radiology. 2004; 233: $830-49$

27. Castellano I, Marchio C, Tomatis M, Ponti A, Casella D, Bianchi S, et al. Micropapillary ductal carcinoma in situ of the breast: an inter-institutional study. Modern pathology : an official journal of the United States and Canadian Academy of Pathology, Inc. 2010; 23: 260-9.

28. Lee YS, Mathew J, Dogan BE, Resetkova E, Huo L, Yang WT. Imaging features of micropapillary DCIS: correlation with clinical and histopathological findings. Academic radiology. 2011; 18: 797-803.

29. Vapiwala N, Hwang WT, Kushner CJ, Schnall MD, Freedman GM, Solin LJ. No impact of breast magnetic resonance imaging on 15-year outcomes in patients with ductal carcinoma in situ or early-stage invasive breast cancer managed with breast conservation therapy. Cancer. 2017; 123: 1324-32.

30. Rauch GM, Hobbs BP, Kuerer HM, Scoggins ME, Benveniste AP, Park YM, et al. Microcalcifications in 1657 Patients with Pure Ductal Carcinoma in situ of the Breast: Correlation with Clinical, Histopathologic, Biologic Features, and Local Recurrence. Ann Surg Oncol. 2016; 23: 482-9.

31. Chagpar A, Yen T, Sahin A, Hunt KK, Whitman GJ, Ames FC, et al. Intraoperative margin assessment reduces reexcision rates in patients with ductal carcinoma in situ treated with breast-conserving surgery. Am J Surg. 2003; 186: 371-7.

32. Carter SA, Lyons GR, Kuerer HM, Bassett RL, Jr., Oates S, Thompson A, et al. Operative and Oncologic Outcomes in 9861 Patients with Operable Breast Cancer: Single-Institution Analysis of Breast Conservation with Oncoplastic Reconstruction. Ann Surg Oncol. 2016; 23: 3190-8.

33. Kuerer HM. Con: Sentinel lymph node biopsy for DCIS. The breast surgeon is not a barber. Ann Surg Oncol. 2007; 14: 1007-8.

34. Mitchell KB, Lin H, Shen Y, Colfry A, Kuerer H, Shaitelman SF, et al. DCIS and axillary nodal evaluation: compliance with national guidelines. BMC Surg. 2017; $17: 12$

35. Francis AM, Haugen CE, Grimes LM, Crow JR, Yi M, Mittendorf EA, et al. Is Sentinel Lymph Node Dissection Warranted for Patients with a Diagnosis of Ductal Carcinoma In situ? Ann Surg Oncol. 2015; 22: 4270-9.

36. Gage I, Schnitt SJ, Nixon AJ, Silver B, Recht A, Troyan SL, et al. Pathologic margin involvement and the risk of recurrence in patients treated with breast-conserving therapy. Cancer. 1996; 78: 1921-8.

37. Huo L. A practical approach to grossing breast specimens. Ann Diagn Pathol. 2011; 15: 291-301.

38. Molina MA, Snell S, Franceschi D, Jorda M, Gomez C, Moffat FL, et al. Breast specimen orientation. Ann Surg Oncol. 2009; 16: 285-8.

39. Toss MS, Pinder SE, Green AR, Thomas J, Morgan DA, Robertson JF, et al. Breast conservation in ductal carcinoma in situ (DCIS): what defines optimal margins? Histopathology. 2017; 70: 681-92.

40. Lester SC, Bose S, Chen YY, Connolly JL, de Baca ME, Fitzgibbons PL, et al. Protocol for the examination of specimens from patients with invasive carcinoma of the breast. Arch Pathol Lab Med. 2009; 133: 1515-38.

41. Hodi Z, Ellis IO, Elston CW, Pinder SE, Donovan G, Macmillan RD, et al. Comparison of margin assessment by radial and shave sections in wide local excision specimens for invasive carcinoma of the breast. Histopathology. 2010; 56: $573-80$

42. Moran MS, Zhao Y, Ma S, Kirova Y, Fourquet A, Chen P, et al. Association of Radiotherapy Boost for Ductal Carcinoma In situ With Local Control After Whole-Breast Radiotherapy. JAMA Oncol. 2017.

43. McCormick B, Winter K, Hudis C, Kuerer HM, Rakovitch E, Smith BL, et al. RTOG 9804: a prospective randomized trial for good-risk ductal carcinoma in situ comparing radiotherapy with observation. J Clin Oncol. 2015; 33: 709-15.

44. Raldow AC, Sher D, Chen AB, Recht A, Punglia RS. Cost Effectiveness of the Oncotype DX DCIS Score for Guiding Treatment of Patients With Ductal Carcinoma In situ. J Clin Oncol. 2016.

45. Manders JB, Kuerer HM, Smith BD, McCluskey C, Farrar WB, Frazier TG, et al. Clinical Utility of the 12-Gene DCIS Score Assay: Impact on Radiotherapy Recommendations for Patients with Ductal Carcinoma In situ. Ann Surg Oncol. 2017; 24: 660-8.

46. Rudloff U, Brogi E, Reiner AS, Goldberg JI, Brockway JP, Wynveen CA, et al. The influence of margin width and volume of disease near margin on benefit of radiation therapy for women with DCIS treated with breast-conserving therapy. Ann Surg. 2010; 251: 583-91.

47. Fisher B, Dignam J, Wolmark N, Wickerham DL, Fisher ER, Mamounas E, et al. Tamoxifen in treatment of intraductal breast cancer: National Surgical Adjuvant Breast and Bowel Project B-24 randomised controlled trial. Lancet (London, England). 1999; 353: 1993-2000.

48. Allred DC, Anderson SJ, Paik S, Wickerham DL, Nagtegaal ID, Swain SM, et al. Adjuvant tamoxifen reduces subsequent breast cancer in women with estrogen receptor-positive ductal carcinoma in situ: a study based on NSABP protocol B-24. J Clin Oncol. 2012; 30: 1268-73.

49. Wapnir IL, Dignam JJ, Fisher B, Mamounas EP, Anderson SJ, Julian TB, et al. Long-term outcomes of invasive ipsilateral breast tumor recurrences after lumpectomy in NSABP B-17 and B-24 randomized clinical trials for DCIS. J Natl Cancer Inst. 2011; 103: 478-88.

50. Cuzick J, Sestak I, Pinder SE, Ellis IO, Forsyth S, Bundred NJ, et al. Effect of tamoxifen and radiotherapy in women with locally excised ductal carcinoma in situ: long-term results from the UK/ANZ DCIS trial. Lancet Oncol. 2011; 12: 21-9.

51. Kuerer HM. Rational individualised selection of adjuvant therapy for ductal carcinoma in situ. Lancet Oncol. 2011; 12: 2-3.
52. Early Breast Cancer Trialists' Collaborative G, Dowsett M, Forbes JF, Bradley $\mathrm{R}$, Ingle J, Aihara $\mathrm{T}$, et al. Aromatase inhibitors versus tamoxifen in early breast cancer: patient-level meta-analysis of the randomised trials. Lancet (London, England). 2015; 386: 1341-52.

53. Margolese RG, Cecchini RS, Julian TB, Ganz PA, Costantino JP, Vallow LA, et al. Anastrozole versus tamoxifen in postmenopausal women with ductal carcinoma in situ undergoing lumpectomy plus radiotherapy (NSABP B-35): a randomised, double-blind, phase 3 clinical trial. Lancet (London, England). 2016; 387: 849-56.

54. Ganz PA, Cecchini RS, Julian TB, Margolese RG, Costantino JP, Vallow LA, et al. Patient-reported outcomes with anastrozole versus tamoxifen for postmenopausal patients with ductal carcinoma in situ treated with lumpectomy plus radiotherapy (NSABP B-35): a randomised, double-blind, phase 3 clinical trial. Lancet (London, England). 2016; 387: 857-65.

55. Forbes JF, Sestak I, Howell A, Bonanni B, Bundred N, Levy C, et al. Anastrozole versus tamoxifen for the prevention of locoregional and contralateral breast cancer in postmenopausal women with locally excised ductal carcinoma in situ (IBIS-II DCIS): a double-blind, randomised controlled trial. Lancet (London, England). 2016; 387: 866-73.

56. Narod SA, Iqbal J, Giannakeas V, Sopik V, Sun P. Breast Cancer Mortality After a Diagnosis of Ductal Carcinoma In situ. JAMA Oncol. 2015; 1: 888-96.

57. Yen TW, Hunt KK, Mirza NQ, Thomas ES, Singletary SE, Babiera GV, et al. Physician recommendations regarding tamoxifen and patient utilization of tamoxifen after surgery for ductal carcinoma in situ. Cancer. 2004; 100: 942-9.

58. Yen TW, Kuerer HM, Ottesen RA, Rouse L, Niland JC, Edge SB, et al. Impact of randomized clinical trial results in the national comprehensive cancer network on the use of tamoxifen after breast surgery for ductal carcinoma in situ. J Clin Oncol. 2007; 25: 3251-8. 